\title{
UNIQUENESS CRITERION FOR A MOMENT PROBLEM
}

\section{A. LENARD}

(Received 2 October 1972)

Communicated by B. Mond

In an article that appeared some years ago in this journal, Takács [1] gave a uniqueness criterion for the solution of the moment problem

$$
\begin{aligned}
B_{r} & =\sum_{k=r}^{\infty}\left(\begin{array}{l}
k \\
r
\end{array}\right) P_{k} \\
r & =0,1,2, \cdots
\end{aligned}
$$

where the $B_{r}$ are given numbers and the $P_{k}$ are sought, $P_{k} \geqq 0, \quad \Sigma_{k} P_{k}=1$. Takács showed that if $B_{r}<\infty$ for all $r$ and

$$
\rho=\limsup _{r \rightarrow \infty}\left(B_{r}\right)^{1 / r}<\infty,
$$

then the solution is unique. In addition, he gave an explicit formula for the solution in this case

$$
P_{k}=\sum_{r=k}^{\infty}\left(\begin{array}{l}
r \\
k
\end{array}\right)(1+q)^{-r-1} \sum_{j=k}^{r}(-1)^{j-k}\left(\begin{array}{l}
r-k \\
j-k
\end{array}\right) q^{r-j} B_{j}
$$

where $q$ is any number satisfying $q \geqq 0$ and $q>\rho^{2}-1$.

The purpose of this note is to give a weaker sufficient condition for uniqueness:

THEOREM. If (1) has a solution and

$$
\sum_{r=1}^{\infty}\left(B_{r}\right)^{-1 / r}=\infty
$$

then the solution is unique.

We note that (4) is satisfied when $B_{r}=O\left(r^{r}\right)$, whereas (2) need not be.

Work partially supported by the Air Force Office of Scientific Research, United States Air Force 
To prove the theorem, we associate with every solution of (1) a generating function

$$
\phi(z)=\sum_{k=0}^{\infty} P_{k} z^{k}
$$

analytic for $|z|<1$. Assume that (1) has more than one solution, and let $P_{k}^{\prime}$, $P_{k}^{\prime \prime}$ be two distinct solutions. Let $\phi^{\prime}$ and $\phi^{\prime \prime}$ be the corresponding generating functions. We write

$$
\psi(z)=\phi^{\prime}(z)-\phi^{\prime \prime}(z)
$$

and proceed to estimate the modulus of $\psi$.

Consider the identity

$$
\begin{aligned}
(1+z)^{k}=1+\left(\begin{array}{l}
k \\
1
\end{array}\right) z+ & \cdots+\left(\begin{array}{c}
k \\
j-1
\end{array}\right) z^{j-1} \\
& +\left(\begin{array}{c}
k \\
j
\end{array}\right) z^{j} j \int_{0}^{1}(1-t)^{j-1}(1+t z)^{k-j} d t .
\end{aligned}
$$

When $|1+z|<1$ and $0 \leqq t \leqq 1$, then $|1+t z| \leqq 1$, therefore

$$
\left|j \int_{0}^{1}(1-t)^{j-1}(1+t z)^{k-j} d t\right| \leqq j \int_{0}(1-t)^{j-1} d t=1 .
$$

Write (7) in the form

$$
z^{k}=\sum_{m=0}^{j-1}\left(\begin{array}{c}
k \\
m
\end{array}\right)(z-1)^{m}+R_{k j}(z)
$$

where, from (8),

$$
\left|R_{k j}(z)\right| \leqq\left(\begin{array}{l}
k \\
j
\end{array}\right)|z-1|^{j}
$$

Now substitute (9) into the right hand side of

$$
\psi(z)=\sum_{k=0}^{\infty}\left(P_{k}^{\prime}-P_{k}^{\prime \prime}\right) z^{k}
$$

Note that the first $j$ terms of (9) contribute nothing since

$$
\sum_{k=0}^{\infty} P_{k}^{\prime}\left(\begin{array}{l}
k \\
m
\end{array}\right)=\sum_{k=0}^{\infty} P_{k}^{\prime \prime}\left(\begin{array}{c}
k \\
m
\end{array}\right)=B_{m}
$$

by hypothesis. Therefore (10) yields

$$
|\psi(z)| \leqq 2 B_{j}|z-1|^{j}
$$

This inequality holds for $|z|<1$ and $j=1,2,3, \cdots$. 
Now we make use of a fact discovered by Carleman [2].

THEOREM. (Carleman). Let $f(z)$ be analytic for $|z|<1$ and not identically zero; let $0<\lambda_{1}<\lambda_{2}<\cdots$ with $\lambda_{n} \rightarrow \infty$; and let $0<\beta_{1}, \beta_{2}, \cdots$. If $f$ satisfies the inequalities

$$
|f(z)| \leqq\left(\beta_{j}|z-1|\right)^{\lambda_{j}}
$$

or $|z|<1$ and $j=1,2, \cdots$ then

$$
\sum_{j=1}^{\infty} \frac{\lambda_{j}-\lambda_{j-1}}{\beta_{j}}<\infty
$$

Carleman proves a slightly different version of this theorem: he works with a half plane instead of an open disk as above. Our statement follows from his in an elementary manner, by performing an appropriate fractional linear transformation.

We now apply Carleman's Theorem with $f(z)=\psi(z), \lambda_{j}=j, \beta_{j}=\left(2 B_{j}\right)^{1 / j}$, and then (13) becomes (14). The conclusion is

$$
\sum_{j=1}^{\infty}\left(B_{j}\right)^{-1 / j}<\infty
$$

and our theorem is proved.

It may be remarked that the derivation of the elegant formula (3) by Takács [1] depends essentially on the analyticity of the generating function $\phi(z)$ in a neighborhood of $z=1$. This is guaranteed by condition (2) but not by the weaker (4). Therefore, under the conditions envisaged by our theorem, the solution of the moment problem (1), while still unique, cannot be given in general by (3).

\section{References}

[1] L. Takács, 'A moment problem', J. Austral. Math. Soc. 5 (1965), 487-490.

[2] T. Carleman, Les Fonctions Quasi-Analytiques (Gauthier-Villars, Paris, 1926) Chapter III.

Institute of Advanced Study

Princeton, New Jersey 08540

U.S.A. 